\title{
Polibutadieno Alto-cis: Estudo Viscosimétrico em Tolueno e Ciclo-hexano
}

\author{
Ivana L. Mello \\ Instituto de Macromoléculas Prof. Eloisa Mano,UFRJ \\ Fernanda M. B. Coutinho, Marcia C. Delpech, Fernanda F. M. Albino, Sânia M. Santos \\ Departamento de Processos Químicos, IQ, UERJ
}

\begin{abstract}
Resumo: Foram realizadas medidas viscosimétricas, em soluções de tolueno e ciclo-hexano, a $30^{\circ} \mathrm{C}$, com polibutadieno alto-cis, sintetizado com catalisadores à base de neodímio. Foram empregadas diferentes equações para determinar os valores de viscosidade intrínseca: Huggins; Kraemer; Martin; e Schulz-Blaschke, por extrapolação gráfica; e SolomonCiuta; Deb-Chanterjee e; novamente, Schulz-Blaschke, por determinação por um único ponto. Os valores de viscosidade intrínseca obtidos pelos dois métodos (extrapolação gráfica e por um único ponto) foram comparados a fim de se verificar a validade da determinação por um único ponto para os sistemas analisados, bem como determinar que equação fosse a mais adequada para esse tipo de cálculo. Foram calculadas as constantes viscosimétricas de Huggins, Kraemer e SchulzBlaschke e foi feita uma análise da qualidade do solvente, levando-se em conta também os valores do parâmetro de solubilidade de Hildebrand dos solventes e do polímero. Foi determinada a distância média quadrática entre as extremidades das cadeias poliméricas por meio da equação de Flory, para tal foram utilizados os dados viscosimétricos obtidos, bem como os pesos moleculares determinados por viscosimetria e por cromatografia de exclusão por tamanho. Verificouse que o método de determinação por um único ponto foi apropriado para o polibutadieno alto-cis, tanto para o cálculo de viscosidade intrínseca quanto de peso molecular. Em tolueno, a equação de Solomon-Ciuta foi a mais adequada, ao passo que a equação de Deb-Chanterjee foi mais apropriada para o polímero em ciclo-hexano. Verificou-se também que o tolueno foi o melhor solvente para o polibutadieno em função dos maiores valores de viscosidade intrínseca e de distância média quadrática entre os extremos da cadeia polimérica. Os valores obtidos para as constantes viscosimétricas confirmaram essa observação.
\end{abstract}

Palavras-chave: Polibutadieno, viscosimetria capilar, determinação por único ponto, distância média quadrática.

\section{High-cis Polybutadiene: Viscometric Study in Toluene and Cyclohexane}

Abstract: Viscometric measurements, in toluene and cyclohexane solutions, at $30^{\circ} \mathrm{C}$, were carried out on high-cis polybutadiene synthesized with neodymium catalysts. Six different equations were employed to calculate intrinsic viscosity values and viscometric constants: Huggins; Kraemer; Martin and Schulz-Blaschke, by graphic extrapolation; and SolomonCiuta; Deb-Chanterjee; and again Schulz-Blaschke, by single point determination. The values obtained for intrinsic viscosity in both solvents and also the molecular weight (in toluene), calculated by applying Mark-Houwink-Sakurada equation, were compared in order to verify the validity of single point determination, which was confirmed. Solomon-Ciuta equation seemed to be suitable for toluene solutions whereas the Deb-Chanterjee equation was adequate for cyclohexane solutions. End-to-end distances of polymeric chains were also determined by applying Flory equation. Based on the higher values of intrinsic viscosity and end-to-end distance of polymeric chains for all samples in toluene, the latter was found to be better solvent than cyclohexane for polybutadiene.

Keywords: Polybutadiene, viscometry, single point determination, end-to-end distance.

\section{Introdução}

Polibutadieno alto-cis é comercialmente produzido pelo processo de polimerização em solução utilizando-se catalisadores Ziegler-Natta. Atualmente, os sistemas catalíticos mais usados são aqueles baseados em lantanídeos, mais especificamente, em neodímio. Tais catali- sadores são mais ativos e mais estereoespecíficos para a polimerização 1,4-cis do que os catalisadores à base de metais de transição (titânio, cobalto e níquel) ${ }^{[1-4]}$.

A caracterização desses polímeros tem sido feita por diversas técnicas. No entanto, há poucas publicações mencionando medidas viscosimétricas em soluções diluídas ${ }^{[5]}$, mesmo sendo a viscosimetria uma técnica que fornece

Autor para correspondência: Fernanda M. B. Coutinho, Departamento de Processos Químicos, IQ, UERJ, Av. Maracanã 524, Pavilhão Haroldo Lisboa da Cunha, CEP: 20559-900, Rio de Janeiro, RJ.E-mail: fern@uerj.br 
resultados importantes e interessantes envolvendo, por exemplo, a qualidade do solvente utilizado, pelos valores de viscosidade intrínseca e das constantes viscosimétricas ${ }^{[6,7]}$, bem como do peso molecular.

A simplicidade do método e dos equipamentos envolvidos faz da viscosimetria um método facilmente aplicável em qualquer laboratório, tanto no meio acadêmico, quanto no industrial. Sendo, no entanto, um método trabalhoso e demorado, quando as determinações são feitas por diluição e os cálculos, por extrapolação gráfica, é interessante que métodos de redução do tempo de análise sejam testados. Para esse último caso, existem equações específicas que podem ser válidas ou não, dependendo do sistema polímero-solventetemperatura.

O efeito observado quando um polímero é adicionado a um solvente é dependente de sua concentração, peso molecular e conformação de sua cadeia. Assim, a viscosimetria é um método apropriado para fornecer informações sobre o tamanho e a forma das macromoléculas em solução. A análise da viscosidade de uma solução polimérica infinitamente diluída resulta na obtenção de parâmetros relativos à cadeia isolada. O principal desses parâmetros é denominado viscosidade intrínseca, definido pela Equação $1^{[8]}$.

$$
[\eta]=\lim _{\mathrm{c} \rightarrow 0}\left[\eta_{\mathrm{sp}} / \mathrm{c}\right]
$$

Onde $\eta_{s p}$ é a viscosidade específica definida pela Equação 2:

$$
\eta_{\mathrm{sp}}=\left(\eta-\eta_{\mathrm{o}}\right) / \eta_{\mathrm{o}}
$$

$\mathrm{Na}$ Equação 2, ๆ é a viscosidade da solução polimérica e $\eta_{\mathrm{o}}$ é a viscosidade do solvente puro. A viscosidade específica está diretamente relacionada à viscosidade relativa, $\eta_{\mathrm{r}}=\eta / \eta_{\mathrm{o}}$, pela Equação 3:

$$
\eta_{\mathrm{sp}}=\eta_{\mathrm{r}}-1
$$

A viscosidade específica determina a contribuição do soluto (polímero) sobre a viscosidade da solução, ou seja, pode ser considerada como o aumento na viscosidade da solução, devido à presença do polímero, em relação à viscosidade do solvente. A viscosidade reduzida (Equação 4) é uma grandeza que expressa a viscosidade específica por unidade de concentração. Quando este parâmetro é extrapolado a uma diluição infinita, obtém-se um valor de viscosidade (viscosidade intrínseca) em condições onde as moléculas podem ser consideradas como isoladas. Assim, a partir da medida da viscosidade específica, é possível obter a viscosidade intrínseca por meio de extrapolação gráfica.

$$
\eta_{\mathrm{red}}=\eta_{\mathrm{sp}} / \mathrm{c}
$$

Diversas equações matemáticas estão disponíveis na lite-

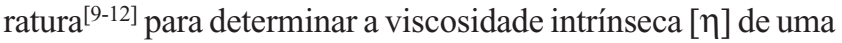
solução polimérica por extrapolação gráfica. As mais utilizadas são as equações de Huggins, Kraemer, Martin e SchulzBlaschke; Equações 5 a 8, respectivamente.

$$
\eta_{\mathrm{sp}} / \mathrm{c}=[\eta]_{\mathrm{h}}+\mathrm{k}_{\mathrm{h}}[\eta]_{\mathrm{h}}^{2} \mathrm{c}
$$

$$
\begin{aligned}
& \ln \eta_{\mathrm{r}} / \mathrm{c}=[\eta]_{\mathrm{k}}-\mathrm{k}_{\mathrm{k}}[\eta]_{\mathrm{k}}^{2} \mathrm{c} \\
& \ln \eta_{\mathrm{sp}} / \mathrm{c}=\ln [\eta]_{\mathrm{m}}+\mathrm{k}_{\mathrm{m}}[\eta]_{\mathrm{m}} \mathrm{c} \\
& \eta_{\mathrm{sp}} / \mathrm{c}=[\eta]_{\mathrm{sb}}+\mathrm{k}_{\mathrm{sb}}\left[\eta_{\mathrm{sb}}\right] \eta_{\mathrm{sp}}
\end{aligned}
$$

onde: $\eta_{\mathrm{r}}=\eta / \eta_{\mathrm{o}}=\mathrm{t} / \mathrm{t}_{\mathrm{o}}=$ viscosidade relativa (razão entre o tempo de escoamento da solução e o tempo de escoamento do solvente puro); $\eta_{\mathrm{sp}}=$ viscosidade específica $\left(\eta_{\mathrm{sp}}=\eta_{\mathrm{r}}-1\right)$ $[\eta]_{\mathrm{h}}=\lim _{\mathrm{c} \rightarrow 0} \eta_{\mathrm{red}}=$ viscosidade intrínseca, relativa à Equação de Huggins

$[\eta]_{\mathrm{k}}=\lim _{\mathrm{c} \rightarrow 0} \eta_{\mathrm{inh}}=$ viscosidade intrínseca, relativa à Equação de Kraemer

$[\eta]_{\mathrm{m}}=\lim _{\mathrm{c} \rightarrow 0} \ln \eta_{\mathrm{red}}=$ viscosidade intrínseca, relativa à Equação de Martin

$[\eta]_{\mathrm{sb}}=\lim _{\mathrm{c} \rightarrow 0} \eta_{\text {red }}=$ viscosidade intrínseca, relativa à Equação de Schulz-Blaschke

$\mathrm{k}_{\mathrm{h}}, \mathrm{k}_{\mathrm{k}}, \mathrm{k}_{\mathrm{m}}$, and $\mathrm{k}_{\mathrm{sb}}=$ constantes de Huggins, Kraemer, Martin e Schulz-Blaschke, respectivamente. Para muitos sistemas poliméricos, o valor de $\mathrm{k}_{\mathrm{sb}}$ é $0,28^{[6,13-17]}$.

Algumas relações têm sido propostas para determinar a viscosidade intrínseca a partir da medida da viscosidade de uma solução diluída por um único ponto. Este método, conforme citado anteriormente, tem a vantagem de ser mais rápido e adequado quando um grande número de amostras precisa ser analisado em um curto espaço de tempo, principalmente em controle de qualidade industrial. Ao contrário das determinações por extrapolação gráfica, este método, dependendo das equações utilizadas, é independente de valores de constantes, o que é uma vantagem.

Combinando as Equações 5 e 6, Solomon e Ciuta ${ }^{[18]}$ chegaram a Equação 9 para a determinação da viscosidade intrínseca.

$$
[\eta]=\left[2\left(\eta_{\mathrm{sp}}-\ln \eta_{\mathrm{r}}\right)\right]^{1 / 2} / \mathrm{c}
$$

A Equação 10 foi proposta por Deb e Chanterjee ${ }^{[19]}$ como uma expressão da viscosidade intrínseca determinada pelo método de um único ponto.

$$
[\eta]=\left(3 \ln \eta_{\mathrm{r}}+3 / 2 \eta_{\mathrm{sp}}^{2}-3 \eta_{\mathrm{sp}}\right) / \mathrm{c}
$$

O uso dessas equações tem sido feito a partir da suposição que $\mathrm{k}_{\mathrm{h}}+\mathrm{k}_{\mathrm{k}}=0,5^{[13]}$.

A viscosidade intrínseca de polímeros, em um dado solvente, aumenta com o peso molecular. Essa relação constitui a base do método viscosimétrico para avaliação do peso molecular de um polímero a partir da equação de MarkHouwink-Sakurada (Equação 11).

$$
[\eta]=K\left(\bar{M}_{v}\right)^{\mathrm{a}}
$$

onde, $\overline{\mathrm{M}}_{\mathrm{v}}$ é o peso molecular viscosimétrico médio, $K$ e $a$ são constantes viscosimétricas que variam em função da natureza do solvente, da temperatura e da estrutura química do polímero $^{[21-24]}$.

O objetivo deste trabalho foi realizar um estudo viscosimétrico de amostras de polibutadieno alto-cis, preparados em nosso laboratório, em dois diferentes solventes: tolueno e ciclo-hexano, pelos métodos de extrapolação gráfica 
e pela determinação a partir de um único ponto. Parâmetros viscosimétricos (viscosidade intrínseca, constantes viscosimétricas e peso molecular viscosimétrico médio), encontrados por ambos os métodos foram avaliados em termos de diferenças percentuais de forma a se verificar a validade do método de determinação por um único ponto para o polímero, nas condições empregadas. Valores da distância média quadrática entre as extremidades das cadeias poliméricas também foram determinados e comparados.

\section{Experimental}

As amostras de polibutadieno alto-cis (BR-1, BR-2, BR3, BR-4 e BR-5) foram preparadas utilizando-se um sistema catalítico Ziegler-Natta à base de neodímio, de acordo com o procedimento descrito em trabalho anterior ${ }^{[25]}$.

A determinação dos pesos moleculares médios foi realizada por cromatografia de exclusão por tamanho (SEC), utilizando o cromatógrafo de permeação em gel (GPC) Waters 600, equipado com injetor automático Waters 717 Autosampler, detector de índice de refração 2410 e colunas de Styragel com limites de exclusão entre 50 e $1 \times 10^{6} \AA$, calibradas com padrões monodispersos de poliestireno (pesos moleculares: $11,6 \times 10^{6} ; 3,80 \times 10^{6} ; 3,15 \times 10^{6} ; 1,10 \times 10^{6} ; 5,70 \times 10^{5} ; 4,00 \times 10^{5}$; $1,70 \times 10^{5} ; 9,92 \times 10^{4} ; 6,21 \times 10^{4} ; 4,20 \times 10^{4} ; 2,42 \times 10^{4} ; 1,23 \times 10^{4}$; $5,45 \times 10^{3} ; 2,40 \times 10^{3} ; 1,47 \times 10^{3}$ ). As medidas foram conduzidas a $30^{\circ} \mathrm{C}$ e as amostras injetadas automaticamente $(1 \mathrm{~mL} / \mathrm{min})$ como soluções a $0,15 \%(\mathrm{~m} / \mathrm{v})$ em tetra-hidrofurano (THF).

A determinação dos parâmetros viscosimétricos foi realizada a $30 \pm 0,1^{\circ} \mathrm{C}$, utilizando-se um viscosímetro capilar Ubbelohde 0B. Foram preparadas soluções a $0,5 \%(\mathrm{~m} / \mathrm{v})$ em tolueno e a $0,25 \%(\mathrm{~m} / \mathrm{v})$ em ciclo-hexano. Pelo método de extrapolação gráfica à diluição infinita, a determinação experimental foi feita pela cronometragem do tempo de escoamento de cinco diluições da solução-mãe polimérica. Nas medidas por um único ponto, escolheu-se a solução de menor concentração para os cálculos. Para a determinação do peso molecular viscosimétrico médio $\left(\overline{\mathrm{M}}_{\mathrm{v}}\right)$, foram utilizadas as constantes $K_{t o l}=30,5 \times 10^{3} \mathrm{~mL} / \mathrm{g}$ e $a_{\text {tol }}=0,725$, para o tolueno ${ }^{[26]}$. Não foi possível calcular $\overline{\mathrm{M}}_{\mathrm{v}}$ quando se utilizou o ciclo-hexano como solvente, pois não foram encontrados na literatura os valores de $K$ e $a$ relativos ao sistema polibutadieno alto-cis / ciclo-hexano a $30^{\circ} \mathrm{C}$.

\section{Resultados e Discussão}

A viscosidade é um fator determinante no escoamento dos fluidos. Esse escoamento, pode ser descrito pela lei de Newton, que determina que a deformação de um fluido cresce contínua e linearmente, qualquer que seja a tensão aplicada. A definição newtoniana clássica para a viscosidade é $\eta=\frac{\tau}{\dot{\gamma}}$. Sendo $\eta$ a viscosidade, $\tau$ a tensão de cisalhamento (força/ área cisalhada) e $\dot{\gamma}$ a taxa de deformação ou gradiente de velocidade.

Se a viscosidade for independente da tensão aplicada ou

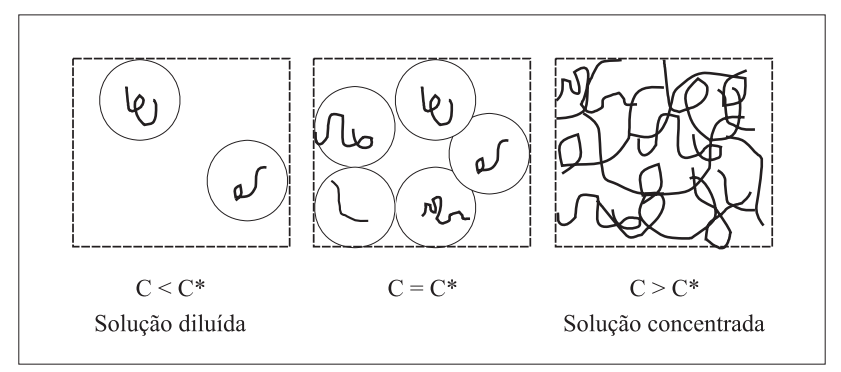

Figura 1. Representação esquemática dos regimes de concentração de uma solução polimérica

do gradiente de velocidade, o fluido é denominado newtoniano. Fluidos não-newtonianos são aqueles cuja viscosidade depende da tensão aplicada ou do gradiente de velocidade. Na prática, o comportamento newtoniano está restrito a moléculas de baixo peso molecular. Porém, soluções poliméricas se comportam como fluidos newtonianos em concentrações próximas a zero (solução diluída).

A viscosidade em sistemas polímero-solvente aumenta rapidamente com o peso molecular do polímero, devido às interações intermoleculares e à formação de entrelaçamentos. Acima de uma concentração crítica, $\mathrm{c}^{*}$ (concentração esta reflete o volume ocupado pela cadeia polimérica isolada) ocorre uma mudança nas propriedades de escoamento devido à formação de entrelaçamentos intermoleculares. Em concentrações mais baixas do que $c^{*}$, as cadeias estão livres para se moverem individualmente, ou seja, as soluções estão em regime diluído ou newtoniano (Figura 1).

A concentração crítica pode ser definida como aquela, a partir da qual, se observa um desvio da linearidade na rela-
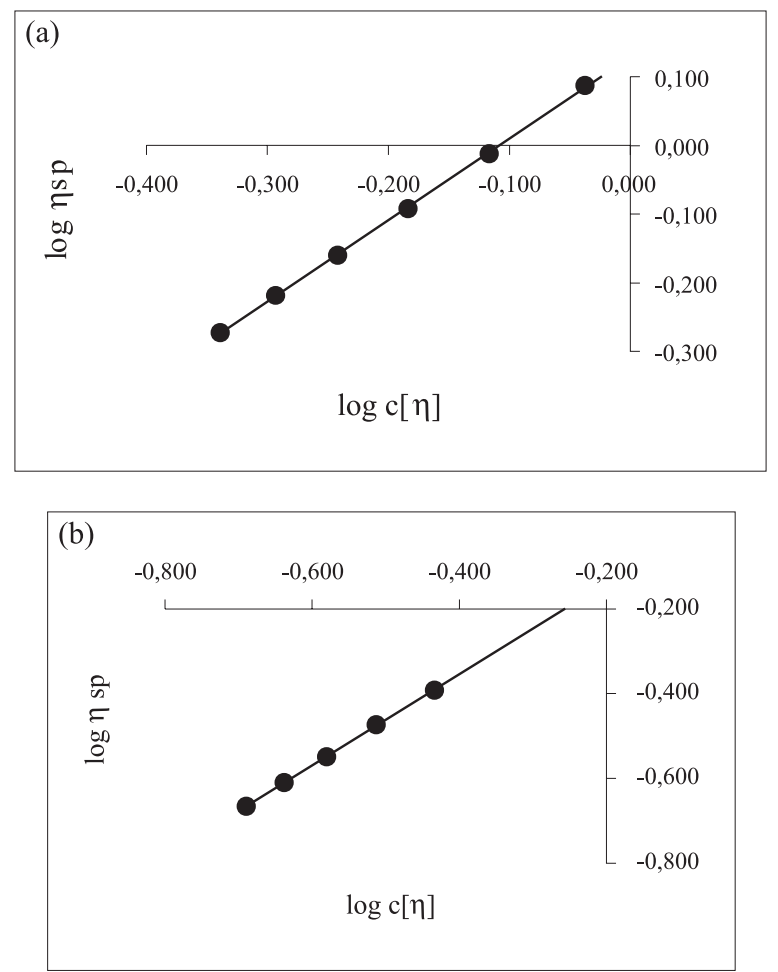

Figura 2. $\log \eta_{\mathrm{sp}}$ versus $\log \mathrm{c}[\eta]$ para o BR-4 em tolueno (a) e ciclohexano (b) 

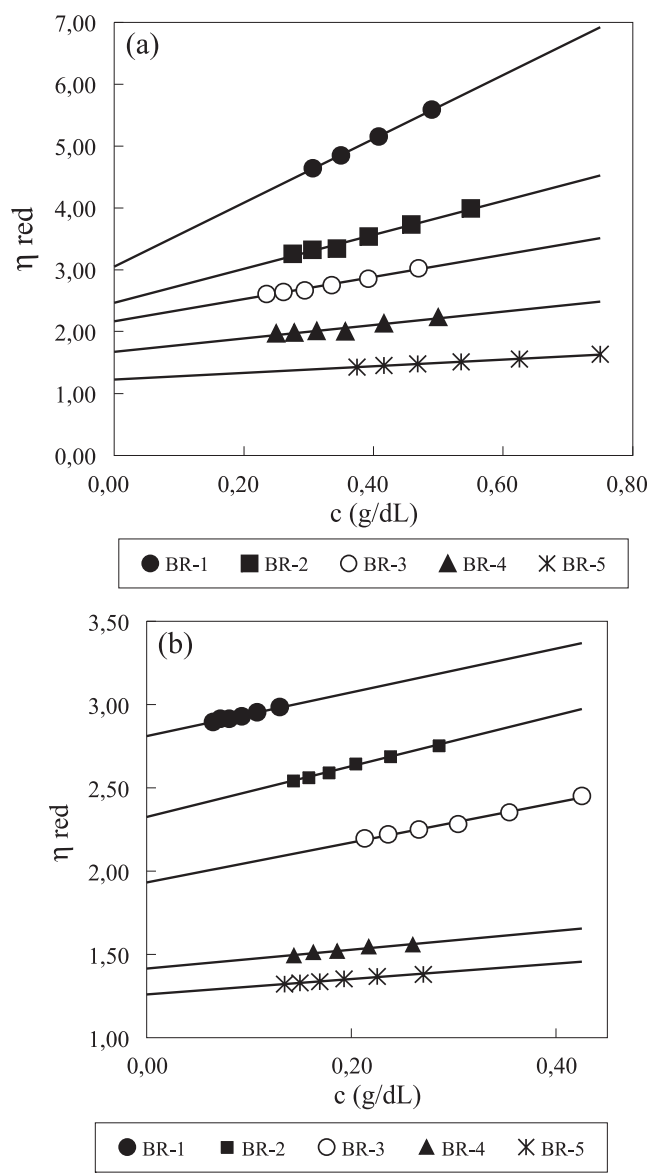

Figura 3. Determinação da viscosidade intrínseca por extrapolação gráfica empregando a equação de Huggins para todas as amostras de polibutadieno alto cis (a) em tolueno e (b) em ciclo-hexano

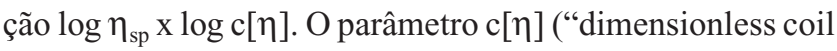
overlap parameter", onde $c$ é a concentração), pode ser definido como o volume total ocupado pelos novelos poliméricos; isto é, c[ๆ] é a medida da fração, em volume, do polímero presente na solução. Esse parâmetro é independente do tipo e do peso molecular do polímero.

A Figura 2 apresenta a relação entre $\log \eta_{\mathrm{sp}}$ e $\log \mathrm{c}[\eta]$ obtida para o BR-4 nos dois solventes estudados, a $30^{\circ} \mathrm{C}$, empregando-se o valor de viscosidade intrínseca fornecido pela equação de Huggins. Foi observada uma relação linear para todas as amostras analisadas, indicando que todas as determinações foram executadas em regime newtoniano.

Os dados viscosimétricos para o polibutadieno alto-cis foram determinados empregando-se as Equações 5 a10. As equações de Huggins (H), Kraemer (K), Martin (M) and Schulz-Blaschke (SB) foram aplicadas no método de determinação da viscosidade intrínseca por extrapolação gráfica.

A Figura 3 ilustra as retas obtidas através da equação de Huggins. Os valores escolhidos para os intervalos de concentração desta Figura garantem que o trabalho foi realizado em regime newtoniano. Tal fato pode ser comprovado pela relação linear observada na Figura 2.

A Tabela 1 apresenta os valores de viscosidade intrínseca relacionado a todas as equações e a Tabela 2 mostra as constantes viscosimétricas obtidas para todas as amostras de polibutadieno alto-cis analisadas.

A constante de Huggins, $k_{h}$, fornece informações sobre as interações entre polímero e solvente. Quanto menor for o seu valor, melhor deverá ser o solvente, ou seja, mais solvatada deverá estar a macromolécula. Resultados experimentais indicam que valores menores que 0,5 são obtidos para soluções

Tabela 1. Valores de viscosidade intrínseca para os sistemas polibutadieno alto-cis tolueno e polibutadieno alto cis/ciclo-hexano, a $30{ }^{\circ} \mathrm{C}$

Tolueno

\begin{tabular}{|c|c|c|c|c|c|c|c|}
\hline \multirow{2}{*}{ Amostra } & \multicolumn{7}{|c|}{ Viscosidade intrínseca $[\eta]$ (dL/g) } \\
\hline & $\mathbf{H}^{\mathbf{a}}$ & $\mathbf{K}^{\mathrm{a}}$ & $\mathbf{M}^{\mathbf{a}}$ & $\mathbf{S B}^{\mathbf{a}}$ & $\mathbf{S B}^{\mathbf{b}}$ & $\mathbf{S C}^{\mathrm{c}}$ & $\mathbf{D C}^{\mathrm{c}}$ \\
\hline BR-1 & 3,051 & 3,211 & 3,405 & 3,629 & 3,320 & 3,386 & 3,670 \\
\hline BR-2 & 2,468 & 2,529 & 2,649 & 2,750 & 2,606 & 2,604 & 2,401 \\
\hline BR-3 & 2,166 & 2,277 & 2,171 & 2,231 & 2,211 & 2,306 & 2,227 \\
\hline BR-4 & 1,674 & 1,687 & 1,717 & 1,744 & 1,731 & 1,716 & 1,777 \\
\hline BR-5 & 1,222 & 1,220 & 1,249 & 1,270 & 1,239 & 1,228 & 1,275 \\
\hline
\end{tabular}

Ciclo-Hexano

\begin{tabular}{|c|c|c|c|c|c|c|c|}
\hline \multirow{2}{*}{ Amostra } & \multicolumn{7}{|c|}{ Viscosidade intrínseca $[\eta](d L / g)$} \\
\hline & $\mathbf{H}^{\mathbf{a}}$ & $\mathbf{K}^{\mathrm{a}}$ & $\mathbf{M}^{\mathbf{a}}$ & $\mathbf{S B}^{\mathbf{a}}$ & $\mathbf{S B}^{\mathrm{b}}$ & $\mathbf{S C}^{\mathrm{c}}$ & $\mathbf{D C}^{\mathrm{c}}$ \\
\hline BR-1 & 2,811 & 2,787 & 2,816 & 2,816 & 2,749 & 2,729 & 2,769 \\
\hline BR-2 & 2,327 & 2,303 & 2,344 & 2,360 & 2,309 & 2,286 & 2,355 \\
\hline BR-3 & 1,931 & 1,917 & 1,959 & 1,984 & 1,942 & 1,923 & 1,983 \\
\hline BR-4 & 1,417 & 1,411 & 1,421 & 1,424 & 1,408 & 1,403 & 1,427 \\
\hline BR-5 & 1,260 & 1,256 & 1,263 & 1,266 & 1,258 & 1,248 & 1,265 \\
\hline
\end{tabular}

${ }^{a}$ Calculados por extrapolação gráfica (H: Huggins; K: Kraemer; M: Martin e SB: Schulz-Blascke

${ }^{\mathrm{b}}$ Calculado através da determinação por um único ponto $\left(\mathrm{k}_{\mathrm{s}} \mathrm{b}=0.28\right)$ (SB: Schulz-Blascke)

${ }^{c}$ Calculados através da determinação por um único ponto (SC: Solomon-Ciuta e DC: Deb-Chanterjee) 
Tabela 2. Constantes viscosimétricas calculadas para o polibutadieno altocis

\begin{tabular}{c|ccccc}
\hline \multicolumn{5}{c}{ Tolueno } \\
\hline Amostra & $\mathbf{k}_{\mathbf{h}}$ & $\mathbf{k}_{\mathbf{k}}$ & $\mathbf{k}_{\mathbf{m}}$ & $\mathbf{k}_{\mathbf{s b}}$ & $\mathbf{k}_{\mathbf{h}}+\mathbf{k}_{\mathbf{k}}$ \\
\hline BR-1 & 0,554 & $-0,103$ & 0,297 & 0,198 & 0,451 \\
BR-2 & 0,450 & $-0,120$ & 0,281 & 0,206 & 0,330 \\
BR-3 & 0,383 & $-0,134$ & 0,287 & 0,230 & 0,249 \\
BR-4 & 0,387 & $-0,136$ & 0,303 & 0,250 & 0,251 \\
BR-5 & 0,362 & $-0,140$ & 0,284 & 0,232 & 0,222 \\
\hline & & Ciclo-Hexano & & \\
\hline Amostra & $\mathbf{k}_{\mathbf{h}}$ & $\mathbf{k}_{\mathbf{k}}$ & $\mathbf{k}_{\mathbf{m}}$ & $\mathbf{k}_{\mathbf{s b}}$ & $\mathbf{k}_{\mathbf{h}}+\mathbf{k}_{\mathbf{k}}$ \\
\hline BR-1 & 0,166 & $-0,408$ & 0,156 & 0,153 & $-0,242$ \\
BR-2 & 0,281 & $-0,177$ & 0,244 & 0,217 & 0,104 \\
BR-3 & 0,322 & $-0,155$ & 0,266 & 0,224 & 0,167 \\
BR-4 & 0,281 & $-0,191$ & 0,258 & 0,242 & 0,090 \\
BR-5 & 0,292 & $-0,187$ & 0,268 & 0,253 & 0,105 \\
\hline
\end{tabular}

poliméricas diluídas em um bom solvente. Já a constante de Kraemer $\left(\mathrm{k}_{\mathrm{k}}\right)$ depende da razão entre o volume hidrodinâmico ocupado por duas moléculas de soluto que interagem entre si e o volume hidrodinâmico ocupado por uma molécula isolada. Quando o coeficiente de Kraemer é negativo significa que há uma boa solvatação da cadeia polimérica, ou seja, há maior interação polímero-solvente ${ }^{[6,14,27]}$.

A Tabela 2 mostra que todas as amostras, tanto em tolueno como em ciclo-hexano, apresentaram $\mathrm{k}_{\mathrm{h}}<0,5$ assim ambos os solventes podem ser considerados como bons solventes para o polibutadieno alto-cis. Esta conclusão pode ser confirmada pelos valores negativos de $\mathrm{k}_{\mathrm{k}}$. Embora, os valores de $\mathrm{k}_{\mathrm{sb}}$, obtidos para as amostras por extrapolação gráfica, sejam diferentes de 0,28 (variando de 0,15 a 0,25) (Tabela 2), o uso deste valor para a determinação por um único ponto produziu valores de $[\eta]_{\mathrm{sb}}$ muito próximos dos obtidos por extrapolação gráfica (Tabela 1).

A Tabela 3 apresenta as diferenças percentuais $(\Delta \%)$ calculadas para os valores de viscosidade intrínseca obtidos pelas equações de Kraemer, Martin, Schulz-Blaschke, Solomon-Ciuta e Deb-Chanterjee, quando comparados com os valores produzidos pela equação de Huggins.

Para os valores obtidos em tolueno, comparando-se as equações de Schulz-Blaschke (SB), Solomon-Ciuta (SC) e Deb-Chanterjee (DC), empregadas no método de determinação por um único ponto, pode-se observar que os valores de viscosidade intrínseca obtidos através da equação SC mostraram uma tendência para menores diferenças percentuais, quando comparados àqueles obtidos pelo método de extrapolação gráfica. A equação SB apresentou também diferenças percentuais reduzidas, mas seu emprego está vinculado ao valor de uma constante $\left(\mathrm{k}_{\mathrm{sb}}=0,28\right)$, o que é uma desvantagem em relação às equações $\mathrm{SC}$ e $\mathrm{DC}$, que
Tabela 3. Diferenças percentuais $(\Delta \%)$ obtidas para valores de viscosidade intrínseca calculadas por extrapolação gráfica (equações de $\operatorname{Kraemer}(\mathrm{K})$, Martin (M) e Schulz-Blaschke (SB)) e por medidas por um único ponto [equações de Schulz-Blaschke (SB), Solomon-Ciuta (SC) e Deb-Chanterjee (DC)], tomando como referência a viscosidade intrínseca de Huggins, [ๆ],

\begin{tabular}{|c|c|c|c|c|c|c|}
\hline \multicolumn{7}{|c|}{$\begin{array}{c}\text { Diferença percentual }(\Delta \%)^{\mathrm{a}} \text { para os valores } \\
\text { de viscosidade intrínseca }\end{array}$} \\
\hline \multirow{3}{*}{ Amostra } & \multicolumn{6}{|c|}{ Tolueno } \\
\hline & \multicolumn{3}{|c|}{ Extrapolação gráfica } & \multicolumn{3}{|c|}{$\begin{array}{l}\text { Medidas por um } \\
\text { único ponto }\end{array}$} \\
\hline & $\mathbf{K}$ & $\mathbf{M}$ & SB & SB & SC & DC \\
\hline BR-1 & 5,24 & 11,60 & 18,95 & 8,82 & 11,00 & 20,29 \\
\hline BR-2 & 2,47 & 7,33 & 11,43 & 5,59 & 5,51 & $-2,71$ \\
\hline BR-3 & 5,12 & 0,23 & 3,00 & 2,08 & 6,46 & 2,82 \\
\hline BR-4 & 0,78 & 2,57 & 4,18 & 3,41 & 2,51 & 6,15 \\
\hline BR-5 & $-0,16$ & 2,21 & 3,93 & 1,39 & 0,49 & 4,34 \\
\hline \multirow{3}{*}{ Amostra } & \multicolumn{6}{|c|}{ Ciclo-Hexano } \\
\hline & \multicolumn{3}{|c|}{ Extrapolação gráfica } & \multicolumn{3}{|c|}{$\begin{array}{l}\text { Medidas por um } \\
\text { único ponto }\end{array}$} \\
\hline & $\mathbf{K}$ & $\mathbf{M}$ & SB & SB & SC & DC \\
\hline BR-1 & $-0,85$ & 0,18 & 0,18 & $-2,21$ & $-2,92$ & $-1,49$ \\
\hline BR-2 & $-1,03$ & 0,73 & 1,42 & $-0,77$ & $-1,76$ & 1,20 \\
\hline BR-3 & $-0,73$ & 1,45 & 2,74 & 0,57 & $-0,41$ & 2,69 \\
\hline BR-4 & $-0,42$ & 0,28 & 0,49 & $-0,64$ & $-0,99$ & 0,71 \\
\hline BR-5 & $-0,32$ & 0,24 & 0,48 & $-0,16$ & $-0,95$ & 0,40 \\
\hline
\end{tabular}

${ }^{\mathrm{a}} \Delta \%=\left[100\left([\eta] /[\eta]_{\mathrm{h}}\right)\right]-100$

independem de qualquer valor. Ao mesmo tempo, é interessante notar que a equação $\mathrm{SB}$, aplicada em um único ponto forneceu diferenças percentuais muito menores do que quando aplicada na extrapolação, indicando que a constante 0,28 é também adequada para o polímero em tolueno. Os resultados indicam que, para o sistema polibutadieno alto-cis/ tolueno, a equação SC parece ser a mais adequada.

Em contrapartida, para o sistema polibutadieno alto-cis/ ciclo-hexano, a equação DC mostrou uma tendência a menores valores quando comparada à equação SC. Novamente, a equação SB, mostrou menores diferenças percentuais no cálculo por um único ponto, empregando $\mathrm{k}_{\mathrm{sb}}=0,28$, em relação à mesma equação aplicada na extrapolação gráfica. $\mathrm{O}$ valor 0,28 parece também ser adequado para o polímero em ciclohexano.

É importante ainda observar que a relação $\mathrm{k}_{\mathrm{h}}+\mathrm{k}_{\mathrm{k}}=0,5$ que, segundo a literatura é uma premissa para que as equações de Solomon-Ciuta e Deb-Chanterjee sejam aplicadas ${ }^{[13]}$, não ocorreu para as amostras analisadas (Tabela 2). No entanto, tanto $[\eta]_{\mathrm{sc}}$ quanto $[\eta]_{\mathrm{dc}}$ apresentaram valores próximos a $[\eta]_{\mathrm{h}},[\eta]_{\mathrm{k}},[\eta]_{\mathrm{m}}$ e $[\eta]_{\mathrm{sb}}$, que foram obtidas por extrapolação gráfica, com diferenças percentuais baixas. Dessa forma a aplicação das equações SC e DC, para o 
Tabela 4. Viscosidade intrínseca de Huggins $[\eta]_{h}$ e valores de peso molecular do polibutadieno alto-cis obtidos por SEC e viscosimetria

\begin{tabular}{|c|c|c|c|c|c|c|c|c|c|c|}
\hline \multirow[b]{2}{*}{ Amostra } & \multicolumn{10}{|c|}{ Tolueno } \\
\hline & $\begin{array}{c}{[\eta] h} \\
(d L / g)\end{array}$ & $\overline{M n}{ }^{1}$ & $\bar{M} w^{1}$ & $\overline{M v_{h}^{2}}$ & $\overline{M v_{k}}{ }^{2}$ & $\bar{M} v_{m}{ }^{2}$ & $\bar{M} \mathbf{v}_{\mathrm{sb}}{ }^{2}$ & $\bar{M} \mathbf{v}_{\mathrm{sb}}{ }^{2}$ & $\bar{M} \mathbf{s c}^{2}$ & $\bar{M} v_{d c}^{2}$ \\
\hline BR-1 & 3,051 & 103000 & 411000 & 329200 & 325300 & 383000 & 418200 & 369900 & 380100 & 424700 \\
\hline BR-2 & 2,468 & 93000 & 340000 & 245400 & 254200 & 271000 & 285200 & 264900 & 264900 & 236300 \\
\hline BR-3 & 2,166 & 57000 & 289000 & 205300 & 205900 & 213800 & 220000 & 213200 & 211200 & 223800 \\
\hline BR-4 & 1,674 & 48000 & 225000 & 143900 & 145400 & 149000 & 152200 & 150600 & 148800 & 156200 \\
\hline BR-5 & 1,222 & 44000 & 223000 & 93200 & 93000 & 96100 & 98300 & 95000 & 93800 & 98800 \\
\hline
\end{tabular}

${ }^{1} \mathrm{SEC}\left(\mathrm{THF}\right.$, a $\left.30^{\circ} \mathrm{C}\right)$

${ }^{2}$ Viscosimetria (Tolueno, a $30{ }^{\circ} \mathrm{C}$ )

polibutadieno alto-cis nos dois solventes analisados, não se mostrou restrita a essa relação.

O peso molecular viscosimétrico médio $\left(\overline{\mathrm{M}}_{\mathrm{v}}\right)$ do polibutadieno alto-cis em tolueno foi calculado a partir da Equação de Mark-Houwink-Sakurada, de acordo com as constantes $K$ e $\alpha$ fornecidas pela literatura para o esse solvente, a $30{ }^{\circ} \mathrm{C}^{[25]}$ (Tabela 4). Não foram encontrados, no entanto, valores correspondentes para o ciclo-hexano.

A viscosidade de soluções diluídas é extremamente afetada pelo peso molecular e pela conformação molecular do polímero. A literatura ${ }^{[27]}$ mostra que um aumento no peso molecular de um polímero provoca um aumento na viscosidade intrínseca, o que é constatado na Tabela 4 para as amostras de polímero nos dois solventes estudados. A Tabela 4 também apresenta uma comparação entre os valores de peso molecular dos polibutadienos obtidos por cromatografia de exclusão por tamanho (SEC) e por viscosimetria, empregando-se os valores de viscosidade intrínseca obtidos a partir das equações 5-10.

Comparando-se os valores de peso molecular viscosimétrico médio ( $\overline{\mathrm{M}} \mathrm{v})$ com os valores de peso molecular ponderal médio ( $\overline{\mathrm{M}} \mathrm{w})$ obtidos por SEC, pode-se observar que, para as amostras com massas mais elevadas, a diferença entre ambos os resultados é menor. Conforme os pesos moleculares diminuem, a diferença entre esses valores vai aumentando especialmente no caso das amostras BR-4 e BR-5. Uma explicação para essa discrepância poderia ser o fato de as constantes $K_{t o l}=30,5 \times 10^{3} \mathrm{~mL} / \mathrm{g}$ e $a_{t o l}=0,725$, obtidas da literatura ${ }^{[25]}$ terem sua aplicação mais restrita para amostras com peso molecular em uma faixa mais elevada. Entretanto, a faixa adequada ${ }^{[26]}$ para a aplicação desses valores é de $5 \times 10^{4}$ a $50 \times 10^{4}$, na qual os valores obtidos por SEC se encaixam. Uma outra possibilidade poderia ser a diversificação nas técnicas de determinação e nas respectivas condições da análise, tais como solventes empregados (THF por SEC, tolueno e ciclo-hexano, por viscosimetria) e valores obtidos por SEC a partir de uma curva de calibração com padrões de poliestireno.

As diferenças percentuais $(\Delta \%)$ obtidas para os valores de peso molecular viscosimétrico médio são mostradas na Tabela 5. Esses valores foram calculados levando-se em conta o valor de $\overline{\mathrm{M}} \mathrm{v}$, determinado pela equação de Huggins como referência. Pode-se notar uma tendência à diminuição nos valores de $\Delta \%$, para todas as amostras, à medida que os pesos moleculares diminuem. Entre os valores de peso molecular obtidos pela determinação por um único ponto, as três equações empregadas (SB, SC e DC) mostraram resultados próximos àqueles obtidos por extrapolação gráfica. A equação de Solomon-Ciuta apresentou novamente a menor diferença percentual, sendo a mais apropriada para ser aplicada em determinações mais rápidas para o sistema polibutadieno alto-cis / tolueno. Dessa forma, verificou-se que o método de determinação por um único ponto foi adequado tanto para o cálculo da viscosidade intrínseca quanto para a determinação do peso molecular.

As dimensões das cadeias poliméricas podem ser medidas, a partir de dados viscosimétricos, por meio da equação de Flory (Equação 12) ${ }^{28}$.

$$
[\eta]=\frac{\phi\left(\overline{\mathrm{h}}^{2}\right)^{3 / 2}}{\mathrm{M}}
$$

onde Mé o peso molecular, $\phi$ é uma constante igual a $2,1 \times 10^{21}$ com $[\eta]$ dada em $100 \mathrm{~mL} / \mathrm{g}$ para polímeros lineares e $\left(\mathrm{h}^{2}\right)^{1 / 2}$ é raiz quadrada da distância média quadrática extremo-a-extremo $(\mathrm{cm})$ da cadeia polimérica.

A Tabela 6 mostra os valores da distância média quadrática extremo-a-extremo de todas as amostras nos dois solventes. Como mencionado anteriormente, durante a descrição da parte experimental, somente para o sistema polibutadieno alto-

Tabela 5. Diferença percentual ( $\Delta \%$ ) obtida por valores de peso molecular viscosimétrico médio

\begin{tabular}{|c|c|c|c|c|c|c|}
\hline \multirow{2}{*}{ Amostras } & \multicolumn{6}{|c|}{ Diferença percentual $(\Delta \%)^{\mathrm{a}}$} \\
\hline & $\overline{\mathbf{M}} \mathbf{v}_{\mathbf{k}}$ & $\overline{\mathbf{M}} \mathbf{v}_{\mathrm{m}}$ & $\overline{\mathbf{M}} \mathbf{v}_{\mathrm{sb}}$ & $\overline{\mathbf{M}} \mathbf{v}_{\mathrm{sb}}$ & $\overline{\mathbf{M}} \mathbf{v}_{\mathrm{sc}}$ & $\overline{\mathbf{M}} \mathbf{v}_{\mathrm{dc}}$ \\
\hline BR-1 & $-1,17$ & 16,36 & 27,06 & 12,38 & 15,48 & 29,03 \\
\hline BR-2 & 3,56 & 10,38 & 16,23 & 7,92 & 7,92 & $-3,73$ \\
\hline BR-3 & 0,32 & 4,16 & 7,13 & 3,87 & 2,87 & 9,02 \\
\hline BR-4 & 1,06 & 3,56 & 5,78 & 4,71 & 3,47 & 8,59 \\
\hline BR-5 & $-0,23$ & 3,06 & 5,44 & 1,93 & 0,67 & 5,97 \\
\hline
\end{tabular}

a $\Delta \%=\left[100\left(\overline{\mathrm{M}}_{\mathrm{v}} / \overline{\mathrm{M}}_{\mathrm{vk}}\right)\right]-100$ 
Tabela 6. Viscosidade intrínseca de Huggins $[\eta]_{h}$ e distância média quadrática $\left(\overline{\mathrm{h}}^{2}\right)$

\begin{tabular}{|c|c|c|c|c|}
\hline \multirow[b]{2}{*}{ Amostra } & \multicolumn{4}{|c|}{ Tolueno } \\
\hline & {$[\eta]_{h}(d L / g)$} & $\begin{array}{c}\left(\overline{\mathbf{h}}^{2}\right) \\
\left(\mathbf{x 1 0 ^ { - 1 1 }}\right)^{*}\end{array}$ & $\begin{array}{c}\left(\overline{\mathbf{h}}^{2}\right) \\
\left(\mathbf{x 1 0 ^ { - 1 1 }}\right)^{* *}\end{array}$ & $\begin{array}{c}\left(\overline{\mathbf{h}}^{2}\right) \\
\left(\mathbf{x 1 0 ^ { - 1 1 }}\right)^{* * * *}\end{array}$ \\
\hline BR-1 & 3,051 & 2,82 & 7,10 & 6,11 \\
\hline BR-2 & 2,468 & 2,29 & 5,43 & 4,36 \\
\hline BR-3 & 2,166 & 1,51 & 4,46 & 3,55 \\
\hline BR-4 & 1,674 & 1,13 & 3,18 & 2,34 \\
\hline BR-5 & 1,222 & 0,87 & 2,56 & 1,43 \\
\hline
\end{tabular}

\begin{tabular}{|c|c|c|c|}
\hline \multirow[b]{2}{*}{ Amostra } & \multicolumn{3}{|c|}{ Ciclo-Hexano } \\
\hline & {$[\eta]_{h}(d L / g)$} & $\begin{array}{c}\left(\overline{\mathbf{h}}^{2}\right) \\
\left(\mathbf{x} 10^{-11}\right)^{*}\end{array}$ & $\begin{array}{c}\left(\overline{\mathbf{h}}^{2}\right) \\
\left(\mathbf{x} 10^{-11}\right)^{* *}\end{array}$ \\
\hline BR-1 & 2,811 & 2,67 & 6,71 \\
\hline BR-2 & 2,327 & 2,20 & 5,21 \\
\hline BR-3 & 1,931 & 1,40 & 4,13 \\
\hline BR-4 & 1,417 & 1,02 & 2,84 \\
\hline BR-5 & 1,260 & 0,89 & 2,61 \\
\hline
\end{tabular}

* Calculada utilizando-se o valor de peso molecular numérico médio $\left(\overline{\mathrm{M}}_{\mathrm{n}}\right)$ ** Calculada utilizando-se o valor de peso molecular ponderal médio $\left(\overline{\mathrm{M}}_{\mathrm{w}}^{\mathrm{n}}\right)$ *** Calculada utilizando-se o valor de peso molecular viscosimétrico médio $(\overline{\mathrm{M}} \mathrm{v})$

cis / tolueno, a $30^{\circ} \mathrm{C}$, foram encontrados na literatura ${ }^{[26]}$ os valores das constantes $K$ e $a$. Assim, somente para esse sistema, foi possível calcular a distância $\left(\bar{h}^{2}\right)$ utilizando-se o valor de peso molecular viscosimétrico médio. Para o sistema polibutadieno alto-cis / ciclo-hexano, calculou-se $\left(\overline{\mathrm{h}}^{2}\right)$ a partir dos valores de peso molecular numérico médio $(\overline{\mathrm{M}} \mathrm{n})$ e peso molecular ponderal médio ( $\overline{\mathrm{M}} \mathrm{w})$, tendo em mente que os valores referentes ao peso molecular viscosimétrico médio ( $\overline{\mathrm{M} v})$ estarão entre esses dois valores.

Pode-se notar que os valores de $\left(\overline{\mathrm{h}}^{2}\right)$ para as amostras em tolueno tendem a ser maiores do que os obtidos em ciclohexano, o que significa que as cadeias poliméricas em tolueno estão mais estendidas. Isso pode ser comprovado pelos valores dos parâmetros de solubilidade de Hildebrand ${ }^{[29]}$ dos solventes e do polibutadieno, todos com estruturas apolares (Tabela 7).

A Tabela 7 mostra que o tolueno é melhor solvente para o polibutadieno, pois seu parâmetro de solubilidade está mais próximo do parâmetro do polímero. Esse solvente tem, portanto, maior poder de solvatação das cadeias poliméricas, ou seja, em tolueno as cadeias estão menos enoveladas do que em ciclo-hexano e, com isso, mais distantes estão as extremi-

Tabela 7. Parâmetros de solubilidade de Hildebrand $(\delta)$

\begin{tabular}{lc}
\hline \multicolumn{1}{c}{ Composto } & $\boldsymbol{\delta}\left(\mathbf{J} / \mathbf{c m}^{3}\right)^{1 / 2}$ \\
\hline Polibutadieno & 17,6 \\
Tolueno & 18,3 \\
Ciclo-hexano & 16,7 \\
\hline
\end{tabular}

dades da cadeia polimérica, isto é, maior é o valor de $\left(\mathrm{h}^{2}\right)$. Outro parâmetro que comprova esse fato é a viscosidade intrínseca. Tendo em vista que os valores de $[\eta]$ encontrados para as amostras em tolueno são maiores do que em ciclohexano, as cadeias poliméricas estão mais estendidas no primeiro, apresentando, portanto, maiores valores de $h^{2}$, e oferecendo, assim, maior resistência ao escoamento.

\section{Conclusões}

O método de determinação da viscosidade intrínseca por um único ponto, utilizando-se as equações de SchulzBlaschke, Solomon-Ciuta e Deb-Chanterjee, mostrou-se apropriado para o polibutadieno alto-cis, tanto em tolueno quanto em ciclo-hexano, a $30^{\circ} \mathrm{C}$. Em tolueno, a equação de Solomon-Ciuta mostrou-se mais apropriada, enquanto que em ciclo-hexano, a equação de Deb-Chanterjee foi a mais adequada. A equação de Schulz-Blaschke, quando aplicada na determinação por um único ponto, apresentou resultados melhores do que quando aplicada na extrapolação gráfica. Os valores de peso molecular para o polibutadieno alto-cis em tolueno, obtidos pelo cálculo por um único ponto, foram muito próximos aos obtidos por extrapolação gráfica, indicando que o primeiro método, que é de determinação muito mais rápida, pode ser empregado para esse sistema. Foi verificado que as constantes de Huggins obtidas para todas as amostras, em ambos os solventes, apresentaram valores inferiores a 0,5 , indicando que, tanto o tolueno quanto o ciclo-hexano são bons solventes para o polibutadieno alto-cis, a $30^{\circ} \mathrm{C}$. Esse resultado foi confirmado pelos valores negativos da constante de Kraemer, que também indicam boa solvatação. Entretanto, comparandose os valores de viscosidade intrínseca, pôde-se notar que o tolueno é ainda um melhor solvente para o polibutadieno do que o ciclo-hexano, pois forneceu os maiores valores de viscosidade intrínseca, para todas as amostras. Esse fato pode ser também confirmado, comparando-se os valores do parâmetro de solubilidade de Hildebrand dos solventes e do polímero e pelos resultados da distância média quadrática extremo-a-extremo da cadeia polimérica.

\section{Agradecimentos}

As autoras agradecem ao Conselho Nacional de Desenvolvimento Científico e Tecnológico (CNPq), à Fundação Carlos Chagas Filho de Amparo à Pesquisa do Estado do Rio de Janeiro (FAPERJ) e à Petroflex Ind. \& Com. pelo suporte financeiro.

\section{Referências Bibliográficas}
1. Mello, I.L.; Coutinho, F. M. B.; Nunes, D. S. S.; Soares, B. G.; Costa, M. A. S.; Maria, L. C. S. - Quím. Nova 27, p.277 (2004)

2. Nickaf, J. B.; Burford, R. P.; Chaplin, R. P. - J. Polym. Sci.: Part A: Polym. Chem., 33, p.1125 (1995) 
3. Lauretti, E.; Miani, B.; Mistrali, F. - Rubber World, may, p.34 (1994)

4. Wilson, D. J. - Makromol. Chem., Macromol. Symp., 66, p.273 (1993)

5. Pires, N. M.; Coutinho, F. M. B.; Costa, M. A. S. - Eur. Polym. J., 40, p.2599 (2004)

6. Delpech, M. C.; Coutinho, F. M. B.; Habibe, M. E. S. Polym. Test., 21, p.411 (2002)

7. Sanches, N. M.; Dias, M. L.; Pacheco, E. B. A. V. - Polym. Test., 24, p.688 (2005)

8. Mitchell, J. R. - "Polysaccharides in food", Ed Mitchell, J.R., London, 51-71 (1979)

9. Huggins, M. L. J. - Am. Chem. Soc. 64, p.2716 (1942)

10. Kraemer, E. O. - Ind. Eng. Chem., 30, p.1200 (1938)

11. Martin, N. B; Tripp, J. B; Shibata, J. H; Schurr, J. M. Biopolymers 18, p.2127 ( 1976)

12. Schulz, G. V; Blaschke, F. J. - Prakt. Chem., 158, p.130 (1941)

13. Abdel-Azim, A. A.; Atta, A. M.; Farahat, M. S.; Boutros, W. Y. - Polymer, 39, p.6827 (1998)

14. Delpech, M. C.; Coutinho, F. M. B.; Habibe, M. E. S. Polym. Test, 21, p.155 (2002)

15. Delpech, M. C.; Oliveira, C. M. F. - Polymer Testing, 24, p. 381 (2005)

16. Khan, H. U.; Gupta, V. K.; Bhargava, G. S. - Polym. Commun., 24, p.191 (1983)

17. Schoff, C. K. - "Polymer Handbook", john Wiley and Sons Inc, New York (1999)
18. Solomon, O.F.; Ciuta, I. Z. - J. Appl. Polym. Sci.; 6, p.683 (1962)

19. Deb P. C. and Chantterjee, S. R. - Indian J. Appl. Chem.; 31, p.121 (1968)

20. Manaresi, P.; Munari, A.; Pilati, F. and Marianucci, E. Eur. Polym. J. 24(6), p. 575 (1988)

21. Robinson, G.; Ross-Murphy, S.; Morris, E. R. - Carbohydr. Res., 107, p.17 (1982)

22. Simionescu, C. I.; Ioan, S.; Simionescu B. C. - Eur. Polym. J., 23, p.69 (1987)

23. Matusinovic, Z.; Rogos M.; Mencer H. J. - Eur. Polym., 41, p.2934 (2005)

24. Moreira, J. C.; Silva, I. F.; Wang, S. H.; Balogh, D. T. Polímeros: Ciência e Tecnologia, 14, p.80, (2004)

25. Mello I. L.; Coutinho F. M. B.; Nunes D. S. S.; Soares B. G.; Costa M. A. S.; Maria L. C. S. - Eur. Polym. J., 40, p.635, (2004)

26. Kurata, M.; Tsunashima, Y. - "Polymer Handbook", John Wiley and Sons Inc, New York, (1999)

27. Tager A. - "Physical chemistry of polymers", Mir Publishers, Moscow (1972)

28. Mousumi, D. S.; Praja P. D.; Anil K. B. - Polymer 40, p.1201 (1999)

29. Krevelen, V.D.W. - "Properties of polymers", Elsevier, Amsterdam (1990)

Enviado: 22/06/05

Reenviado: 26/10/05

Aprovado: 29/11/05 